\title{
Primary cerebellar B cell lymphoma: A case report
}

\author{
Anupam Datta, Arunima Gupta, Krishnangshu Bhanja Choudhury, \\ Aruj Dhyani, Anup Majumdar
}

\begin{abstract}
Introduction: Primary central nervous system lymphoma (PCNSL) is usually a diffuse large B cell non-Hodgkin lymphoma that originates in the brain, spinal cord, leptomeninges or eyes. Primary central nervous system lymphoma is a rare malignant tumor comprising less than $3 \%$ of all primary brain tumors. The PCNSL in immunocompetent patients is most commonly supratentorial, found adjacent to the ventricular surfaces and in deep white matter and subcortical structures, such as the basal ganglia, thalamus and corpus callosum. The lesion is single in $60-70 \%$ of patients. Lesions are located in the hemispheres (38\%), thalamus/basal ganglia (16\%), corpus callosum (14\%), periventricular region (12\%) and cerebellum (9\%). Herein, we report an extremely rare case of a primary CNS B cell lymphoma involving cerebellum. Case Report: A 55-year-old female was presented with vomiting, ataxia and disorientation. T2-
\end{abstract}

Anupam Datta ${ }^{1}$, Arunima Gupta ${ }^{1}$, Krishnangshu Bhanja Choudhury $^{2}$, Aruj Dhyani ${ }^{1}$, Anup Majumdar ${ }^{3}$

Affiliations: ${ }^{1}$ Post Graduate trainee, Radiotherapy, Junior Resident, Department of Radiotherapy, Institute of Post graduate Medical Education and Research, Kolkata-700020, West Bengal, India; ${ }^{2} \mathrm{MD}$, Radiotherapy. RMO-cum-Clinical tutor, Department of Radiotherapy, R.G.Kar Medical College and Hospital, Kolkata -700004, West Bengal, India; ${ }^{3} \mathrm{MD}$, Radiotherapy, Professor and Head, Department of Radiotherapy, Institute of Post graduate Medical Education and Research, Kolkata-700020, West Bengal, India.

Corresponding Author: Krishnangshu Bhanja Choudhury, Designation: RMO-cum-Clinical tutor, Department of Radiotherapy, R.G.Kar Medical College and Hospital, Kolkata -700004, West Bengal, India; Address: C-11/4, Green Tower, Golf Green, Kolkata -700095, West Bengal; Ph: +919830932869; E-mail: krishnangshuchoudhury@gmail.com

Received: 30 July 2012

Accepted: 06 March 2013

Published: 01 September 2013 weighted axial, T1-weighted and gadoliniumenhanced T1-weighted axial magnetic resonance imaging scans show a contrast enhancing mass with peritumoral edema in the right cerebellar hemisphere. Excision biopsy was suggestive of non-Hodgkin lymphoma. Diagnosis was confirmed with immunohistochemistry favoring non-Hodgkin lymphoma of diffuse large $B$ cell type. This patient received cytotoxic therapy with carmustine $200 \mathrm{mg}$ IV 6 weekly 3 cycles followed by external beam radiation therapy $30 \mathrm{~Gy}$. No recurrence was noticed in one year follow-up. Conclusion: Primary B cell lymphoma limited to the CNS is exceedingly rare in the middle aged immunocompetent individual. Little is known regarding etiologic factors, optimal management and prognosis.

Keywords: Non-Hodgkin lymphoma, Carmustine, B-cell lymphoma, Cerebellum

$$
* * * * * * * * *
$$

Datta A, Gupta A, Choudhury KB, Dhyani A, Majumdar A. Primary cerebellar B cell lymphoma: A case report. International Journal of Case Reports and Images 2013;4(9):498-501.

$* * * * * * * * *$

doi:10.5348/ijcri-2013-09-365-CR-9

\section{INTRODUCTION}

Primary central nervous system lymphoma (PCNSL) is usually a diffuse large B cell non-Hodgkin lymphoma that originates in the brain, spinal cord, leptomeninges or eyes. The PCNSL is a rare malignant tumor representing $3 \%$ of intracranial neoplasms and $4-6 \%$ of extra nodal lymphomas with a yearly incidence of 0.5 case per 100,000 people and with a median survival, if untreated, of 1.5-3.3 
months. It is rare in immunocompetent patients. Median age at diagnosis is 60-65 years. The lesion is single in 60$70 \%$ of patients. Lesions are located in the hemispheres (38\%), thalamus/basal ganglia (16\%), corpus callosum (14\%), periventricular region (12\%) and cerebellum (9\%). We report unusual primary cerebellar B cell lymphoma in a middle aged immunocompetent female patient.

\section{CASE REPORT}

A 55-year-old female was admitted in emergency department with symptoms of ataxia and disorientation of 1 week duration. She was suffering from headache and vomiting for last four months. The headache was diffuse, constant, throbbing and associated with episodic nausea and vomiting. Concurrently, she had a weight loss of $11 \mathrm{~kg}$, daily fever and chills over the same duration. Clinical examination revealed slurred speech, finger to nose ataxia worse on the right side and truncal ataxia, with no other significant neurological deficits. A computed tomography (CT) scan of the brain revealed a solitary, well circumscribed, cystic, ring enhancing mass in the right cerebellar hemisphere with surrounding edema. T2weighted axial, T1-weighted and gadolinium- enhanced T1- weighted axial magnetic resonance imaging (MRI) scans of brain showed a contrast enhancing mass with peritumoral edema in the right cerebellar hemisphere (Figure 1). Complete hemogram, liver function and renal function tests along with serum electrolytes were normal. Skiagram of chest and ultrasonography of abdomen were normal. She was seronegative for HIV 1 and 2, HBsAg and anti-HCV antibodies. Intravenous dexamethasone was initiated and a subtotal resection of the cerebellar lesion was performed. Hematoxylin and eosin preparation revealed a malignant round cell tumor probably non-Hodgkin lymphoma (Figure 2A-C). Diagnosis was confirmed with immunohistochemistry positivity for CD20 and CD10 (dim) and tumor stained immunonegative for $\mathrm{CD}_{3}, \mathrm{CD}_{5}$ and CD23. The Mib-1 labeling index was approximately 60-70\%. Therefore, giving the impression of non-Hodgkin lymphoma (NHL) of diffuse large B cell type. With this biopsy report further investigations of CT scan of abdomen and bone marrow examination were done; all studies were normal. After three weeks of surgery cerebrospinal fluid (CSF) analysis

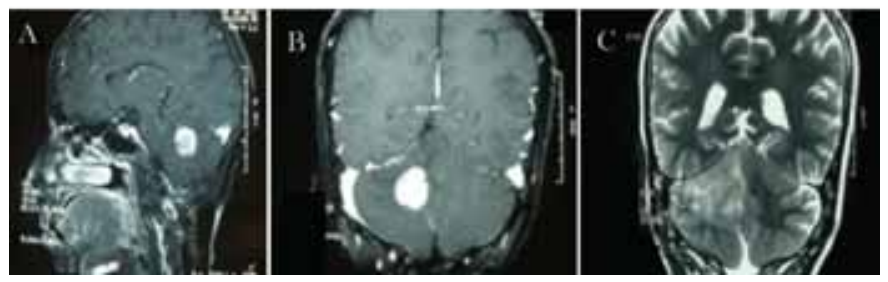

Figure 1: Magnetic resonance imaging scan (A) T2-weighted axial, (B) T1-weighted (C) and Gadolinium-enhanced T1weighted axial. Magnetic resonance imaging scan showing a contrast enhancing mass with peritumoral edema in the right cerebellar hemisphere. showed normal glucose and protein levels, and 2 cells/ $\mathrm{mm}^{3}$, all of which were lymphocytes. She was diagnosed as primary CNS non-Hodgkin lymphoma and went on to receive cytotoxic therapy with carmustine $200 \mathrm{mg}$ IV 6 weekly three cycles followed by external beem radiation therapy (EBRT) 30 Gy. No recurrence was noticed in one year follow-up. Post treatment CT scan of the brain revealed no abnormality (Figure 3 ).
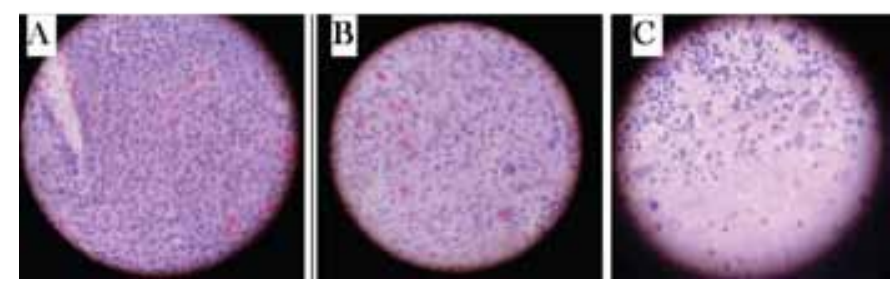

Figure 2: (A , B, C) Hematoxylin-eosin stained biopsy sections showing normal cerebellar tissue and a tumor composed of sheets of round or oval cells with hyperchromatic nuclei. Mitotic figures seen, 40x and 100x.

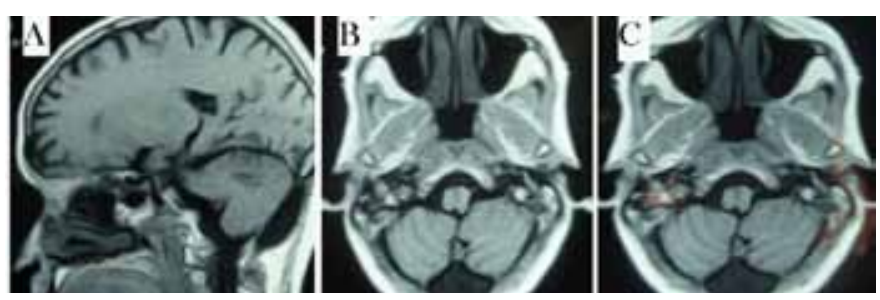

Figure 3: Follow-up magnetic resonance imaging scan after treatment. (A) T2-weighted axial, (B) T1-weighted axial and (C) Gadolinium-enhanced T1-weighted axial. There is no evidence of residual or local recurrence.

\section{DISCUSSION}

The PCNSL is defined as lymphoma limited to the cranial-spinal axis without systemic disease [1]. In the past, PCNSL was considered a rare disorder, accounting for $1-2 \%$ of all cases of non-Hodgkin lymphoma and fewer than $5 \%$ of all cases of primary intracranial neoplasm [2], just $13 \%$ of these arise in the posterior fossa. The PCNSL now represents around $3 \%$ of intracranial neoplasms and 4-6\% of extranodal lymphomas with a yearly incidence of 0.5 case per 100,000 people.

An increasing incidence of this disease has been seen among patients with acquired immunodeficiency syndrome (AIDS) and among other immunocompromised persons. Theincidence of PCNSLintheimmunocompetent population has been reported to have increased more than 10 -fold from 2.5 cases to 30 cases per 10 million populations [3]. The cause for the increase in incidence of this disease in the immunocompetent population is unknown. Two theories have been proposed. Lymphoma develops within a focus of inflammation in the CNS, as may occur in other organ systems such as the gastrointestinal tract. Another possible explanation is 
that the cancerous lymphocytes develop elsewhere in the body, but acquire a receptor on their surface that draws them to a signal expressed only inside the CNS. The CNS is an immunologic sanctuary site rendering the neoplastic lymphocytes which may be systemically eradicated by an intact immune system, find relative protection within the central nervous system. In patients with AIDS, infection with the Epstein-Barr virus, which also causes infectious mononucleosis, is probably an important trigger for PCNSL, although the reason for this is not understood.

The natural history of this disorder differs between patients with AIDS and those without AIDS. The CT scans may show ring enhancement in 50\% of AIDS patients while patients without AIDS almost always show only homogeneous enhancement [1]. Both groups do equally poorly without therapy (1-3 months mean survival), but the overall survival for treated patients is much better for patients without AIDS (18.9 months) than for those with AIDS (2.6 months) [1, 4].

Although more than $95 \%$ of patients with PCNSL are lymphoma of B cell origin, 45 patients with CNS lymphoma of $\mathrm{T}$ cell origin showed no difference in presentation or outcome in a retrospective series with data collected from 12 cancer centers [5]. Almost all are aggressive neoplasms of the diffuse large B-cell type. In a retrospective case series derived from 18 cancer centers in five countries of 40 patients with low-grade primary CNS lymphoma, a better long-term outcome was shown (7-year median survival) than is associated with the usual aggressive CNS lymphoma [6]. Anecdotal cases of primary CNS Hodgkin lymphoma have also been reported [7].

The PCNSL in immunocompetent patients is most commonly found adjacent to the ventricular surfaces and in deep white matter and subcortical structures, such as the basal ganglia, thalamus and corpus callosum. Most of these tumors are supratentorial. In one series, more than $70 \%$ of tumors were in a cerebral hemisphere and periventricular location (usually involving the corpus callosum or basal ganglia). Approximately, 50-70\% of patients with this disease presented with solitary lesions, whereas the remainder had multifocal disease. These lesions characteristically enhance homogeneously with the administration of contrast agents [8]. The incidence of cerebellar involvement in one study in 2005 (16 of 170 lesions) was similar to the findings of Lanfermann et al. (15 of 174 lesions) [9, 10]. The exact incidence of cerebellar PCNSL in a middle aged individuals remains unclear inspite extensive literature search. The differential diagnosis of PCNSL includes gliomas, metastatic tumors, demyelinating disorders, subacute infarcts, and space-occupying lesions due to an infectious etiology.

The optimal treatment for includes radiation, high dose methotrexate, or more complicated regimen of de Angelis. Surgery other biopsy provides no therapeutic benefit because of the depth and diffuse nature of the tumor and should be reserved for the rare patient with neurological deterioration due to brain herniation. Antimetabolites such as methotrexate (MTX) and cytarabine (ara-C) remain most common chemotherapeutic agents used in PCNSL regimens. The MTX doses in excess of $1 \mathrm{gm} / \mathrm{m}^{2}$ result in tumoricidal levels in the brain parenchyma and doses $3 \mathrm{~g} / \mathrm{m}^{2}$ yield tumoricidal levels in the CSF. However, MTX administration requires intensive monitoring with serum MTX level measurements. Other drugs used are carmustine, temozolomide, procarbazine and vincristine. Whole-brain radiation therapy alone is insufficient for durable tumor control and is associated with a high-risk of neurotoxicity in patients over 60 years of age. The initial response to radiation therapy in immunocompetent patients is excellent, often resulting in complete resolution of radiographic abnormalities. Nevertheless, the duration of response is short and median survival duration with radiation therapy alone averages only 18 months. Relapse in patients with parenchymal disease is usually within the brain, though leptomeningeal, vitreous and, rarely, systemic recurrences are reported.

The prognosis of nonselected patients with PCNSL is comparable to that of glioblastoma multiforme patients, with a 5-year survival rate after conventional chemotherapy and radiotherapy (RT) usually at less than 10\% [11]. Unfavorable prognostic factors for survival are more than 60 years of age, WHO performance status greater than 2, poor neurologic function, elevated serum lactate dehydrogenase, extensive tumor spread, CSF protein level greater than $0.6 \mathrm{~g} / \mathrm{L}$, corticosteroid dependence, and absence of chemotherapy administration [12-14].

\section{CONCLUSION}

Primary B cell lymphoma is limited to the central nervous system, more so to cerebellum. It is rare in middle aged, immunocompetent individual. Little is known regarding etiologic factors, optimal management, and prognosis.

\section{$* * * * * * * * *$}

\section{Author Contributions}

Anupam Datta -Conception and design, Drafting the article, Critical revision of the article, Final approval of the version to be published

Arunima Gupta - Conception and design, Acquisition of data, Analysis and interpretation of data, Critical revision of the article, Final approval of the version to be published Krishnangshu Bhanja Choudhury - Conception and design, Acquisition of data, Analysis and interpretation of data, Drafting the article, Critical revision of the article, Final approval of the version to be published

Aruj Dhyani - Conception and design, Drafting the article, Critical revision of the article, Final approval of the version to be published

Anup Majumdar - Conception and design, Drafting the 
article, Critical revision of the article, Final approval of the version to be published

\section{Guarantor}

The corresponding author is the guarantor of submission.

\section{Conflict of Interest}

Authors declare no conflict of interest.

\section{Copyright}

(C) Anupam Datta et al. 2013; This article is distributed under the terms of Creative Commons attribution 3.0 License which permits unrestricted use, distribution and reproduction in any means provided the original authors and original publisher are properly credited. (Please see www.ijcasereportsandimages.com/copyright-policy.php for more information.)

\section{REFERENCES}

1. Fine HA, Mayer RJ. Primary central nervous system lymphoma. Ann Intern Med 1993;119(11):1093-104.

2. Jellinger K, Radaskiewicz TH, Slowik F. Primary malignant lymphomas of the central nervous system in man. Acta Neuropathol Suppl 1975;Suppl 6:95102.

3. Corn BW, Marcus SM, Topham A, Hauck W, Curran WJ Jr. Will primary central nervous system lymphoma be the most frequent brain tumor diagnosed in the year 2000? Cancer 1997;79(12):2409-13.

4. Nasir S, DeAngelis LM. Update on the management of primary CNS lymphoma. Oncology 2000;14(2):22834.

5. Shenkier TN, Blay JY, O'Neill BP, et al. Primary CNS lymphoma of T-cell origin: a descriptive analysis from the international primary CNS lymphoma collaborative group. J Clin Oncol 2005;23(10):2233-9.

6. Jahnke K, Korfel A, O’Neill BP, et al. International study on low-grade primary central nervous system lymphoma. Ann Neurol 2006;59(5):755-62.

7. Gerstner ER, Abrey LE, Schiff D, et al. CNS Hodgkin lymphoma. Blood 2008;112(5):1658-61.

8. Moradi A, Tajedini A, Mehrabian A, et al. Clinicopathogical features of primary central nervous system lymphoma. Neurosciences 2006;11(4):284-8.

9. Lanfermann H, Heindel W, Schaper J, et al. CT and MR imaging in primary cerebral non-Hodgkin's lymphoma. Acta Radiol 1997;38(2):259-67.

10. Küker W, Nägele T, Korfel A, et al. Primary central nervous system lymphomas (PCNSL): MRI features at presentation in 100 patients. Journal of NeuroOncology 2005;72(2):169-77.

11. Blay JY, Lasset C, Carrie C, et al. Multivariate analysis of prognostic factors with non HIV-related Primary cerebral lymphoma: A proposal for a prognostic scoring. Br J Cancer 1993;67(5):1136-41.

12. Michalski JM, Garcia DM, Kase E, Grigsby PW, Simpson JR. Primary central nervous system lymphoma: Analysis of Prognostic variables and patterns of treatment failure. Radiology 1990;176(3):855-60.

13. Blay JY, Conroy T, Chevreau C, et al. High-dose methotrexate for the treatment of primary central nervous system lymphomas: Analysis of survival and late neurologic toxicity in a retrospective series. J Clin Oncol 1998;16(3):864-71.

14. Nelson DF, Martz KL, Bonner H, et al. Non-Hodgkin's lymphoma of the brain: Can high-dose, large volume radiation therapy improve survival? Report on a prospective trial by the Radiation Therapy Oncology Group (RTOG)-RTOG 8315. Int J Radiat Oncol Biol Phys 1992;23(1):9-17.
Access full text article on other devices

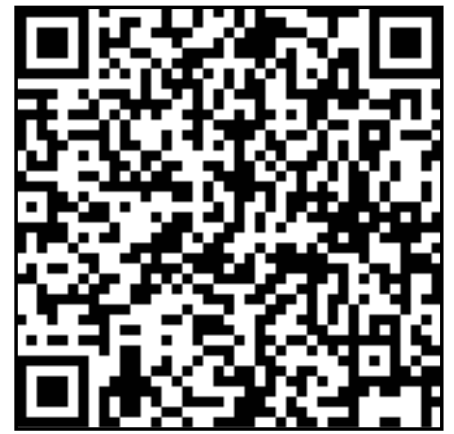

Access PDF of article on other devices

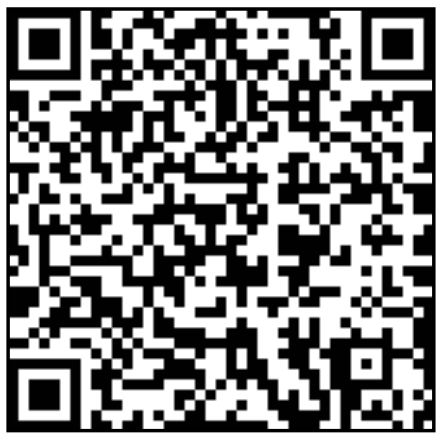

